\title{
REMARKS ON THE REDUCTION THEORY OF VON NEUMANN ALGEBRAS
}

\author{
MASAMICHI TAKESAKI ${ }^{1}$
}

In [7, Problem 4, p. 3.55], S. Sakai proposed the following question: If any component of a measurable field $\gamma \mapsto \mathfrak{T}(\gamma)$ of von Neumann algebras over $(\Gamma, \mu)$ is isomorphic to some fixed von Neumann algebra $\mathfrak{T T}_{0}$ with separable preduar, where $\Gamma$ means a locally compact Hausdorff space and $\mu$ a positive Radon measure on $\Gamma$, is it true that the direct integral $\int_{\Gamma_{0}}^{\oplus} \mathfrak{T}(\gamma) d \mu(\gamma)$ is isomorphic to the tensor product $Q \otimes \mathbb{M}_{0}$ of the associated diagonal algebra ${ }^{n} Q=L^{\infty}(\Gamma, \mu)$ and $\mathfrak{T M}_{0}$ ? If $\Gamma$ satisfies the second countability axiom, then one can easily settle the above problem affirmatively as an application of [1, Proposition 4, p. 187]. However, in the case that the countability assumption of $\Gamma$ is dropped, J. Dixmier proposed the similar problem in $[1$, p. 175]. Namely, roughly speaking, if $\gamma \mapsto y_{i}(\gamma)$ is a measurable operator field over $(\Gamma, \mu)$ for each $i \in I$ and there exists a family of bounded operators $\left\{x_{i}\right\}_{i \in I}$ on a Hilbert space and an isometric operator $u(\gamma)$ for each $\gamma$ such that $u(\gamma) x_{i} u(\gamma)^{-1}=y_{i}(\gamma)$ for every $\gamma \in \Gamma$ and $i \in I$, does there exist an isometry which transforms $1 \otimes x_{i}$ to $\int_{\Gamma}^{\oplus} y_{i}(\gamma) d \mu(\gamma)$ for every $i \in I$ ?

In this paper, we shall settle the above two related questions affirmatively.

Let $\Gamma$ be a locally compact Hausdorff space and $\mu$ a positive Radon measure on $\Gamma$. Let $\gamma \mapsto H(\gamma)$ be a measurable field of Hilbert spaces over $\Gamma$ in the sense of [1]. Let $H_{0}$ be a separable Hilbert space. If $H(\gamma)=H_{0}$ for every $\gamma \in \Gamma$ and the measurable vector fields are measurable $H_{0}$-valued functions over $\Gamma$, then $\gamma \mapsto H(\gamma)$ is called a constant field corresponding to $H_{0}$. If $\gamma \mapsto \operatorname{dim}, H(\gamma)$ is constant, then the measurable field $\gamma \mapsto H(\gamma)$ of Hilbert spaces is isomorphic to a constant measurable field corresponding to some Hilbert space $H_{0}$. Namely, there exist a Hilbert space $H_{0}$ and isometry $u(\gamma)$ of $H(\gamma)$ onto $H_{0}$ for every $\gamma \in \Gamma$ such that $\gamma \mapsto \xi(\gamma) \in H(\gamma)$ is a measurable vector field if and only if $u(\gamma) \xi(\gamma) \in H_{0}$ is a measurable $H_{0}$-valued function over $\Gamma$. Putting $(u \xi)(\gamma)=u(\gamma) \xi(\gamma)$ for every $\xi=\int_{\Gamma}^{\oplus} \xi(\gamma) d \mu(\gamma) \in H$ $=\int_{\Gamma}^{\oplus} H(\gamma) d \mu(\gamma), u$ is an isometry of $H$ onto the Hilbert space $L_{H_{0}}^{2}(\Gamma, \mu)$ of all square integrable $H_{0}$-valued functions over $\Gamma$. Therefore, $H$ is isomorphic to the tensor product $L^{2}(\Gamma, \mu) \otimes H_{0}$.

Received by the editors October 23, 1967 and, in revised form, December 4, 1967.

1 The author is partially supported by Sakkokai Foundation. 
TheOREM 1. Let $H_{0}$ be a separable Hilbert space and $\left\{x_{i}\right\}_{i \in I}$ a family of bounded operators on $H_{0}$. Let $\gamma \mapsto H(\gamma)$ be a measurable field of Hilbert spaces over $\Gamma$,

$$
H=\int_{\Gamma}^{\oplus} H(\gamma) d \mu(\gamma),
$$

and, for each $i \in I, \gamma \mapsto y_{i}(\gamma)$ is a measurable essentially bounded operator field over $\Gamma$ with the direct integral

$$
y_{i}=\int_{\mathbf{r}}^{\oplus} y_{i}(\gamma) d \mu(\gamma) \in \Theta(H) .
$$

Suppose that there exists, for every $\gamma \in \Gamma$, an.isometry $u(\gamma)$ of $H_{0}$ onto $H(\gamma)$ such that

$$
y_{i}(\gamma)=\dot{u}(\gamma) x_{i} u(\gamma)^{-1}, \quad i \in I .
$$

Then there exists an isometry of $L^{2}(\Gamma, \mu) \otimes H_{0}$ onto $H$ which transforms, for every $i \in I, 1 \otimes x_{i}$ into $y_{i}$.

Proof. By the above consideration, we may assume that $\gamma \mapsto H(\gamma)$ is a constant field corresponding to $H_{0}$. Then $\gamma \mapsto y_{i}(\gamma)$ is a $B\left(H_{0}\right)$ valued measurable function over $\Gamma$, where the strong operator topology is considered in the full operator algebra $B\left(H_{0}\right)$, so that $\gamma \mapsto u(\gamma) x_{i} u(\gamma)^{-1}$ is measurable for every $i \in I$. Let $\Re_{0}$ be the von Neumann algebra generated by $\left\{x_{i}\right\}_{i \in I}$. Let $\mathscr{r}$ be the set of all bounded operators $x$ on $H_{0}$ with the property; $\gamma \mapsto u(\gamma) x u(\gamma)^{-1}$ is measurable. Then $\mathscr{N}$ is a ${ }^{*}$-algebra containing $\left\{x_{i}\right\}_{i \in I}$. If $\left\{a_{n}\right\}$ is a bounded sequence in $\mathscr{X}$ strongly converging to $a_{0} \in B\left(H_{0}\right)$, then $u(\gamma) a_{n} u(\gamma)^{-1}$ also converges strongly to $u(\gamma) a_{0} u(\gamma)^{-1}$, so that $\gamma \mapsto u(\gamma) a_{0} u(\gamma)^{-1}$ is measurable. By the separability of $H_{0}$, the strong operator topology in bounded parts of $\Theta\left(H_{0}\right)$ is metrizable, so that $\mathscr{T}$ is strongly closed. Therefore, $\mathscr{N}$ is a von Neumann algebra containing $\left\{x_{i}\right\}_{i \in I}$, and $\mathscr{N}$ contains $\mathfrak{M}_{0}$. Thus, for every $x \in \Re_{0}, \gamma \mapsto u(\gamma) x u(\gamma)^{-1}$ is measurable. Again by the separability of $H_{0}$, there exists a separable strongly dense $C^{*}$-subalgebra $A$ of $\mathfrak{M}_{0}$. Let $\operatorname{Rep}\left(A: H_{0}\right)$ denote the space of all representations of $A$ on $H_{0}$. Then $\operatorname{Rep}\left(A: H_{0}\right)$ is a Polish space with respect to the simple convergence topology, where the strong operator topology is considered in $B\left(H_{0}\right)$. Let $\mathfrak{U}$ denote the group of all unitary operators on $H_{0}$, which is a Polish group with respect to the strong operator topology. For each $u \in \mathfrak{U}$, define the action of $u$ on $\pi \in \operatorname{Rep}\left(A: H_{0}\right)$ by $(u \pi)(x)=u \pi(x) u^{-1}$ for every $x \in A$. Then $\mathfrak{U}$ becomes a topological transformation group of $\operatorname{Rep}\left(A: H_{0}\right)$. Let $\pi_{0}$ be the identity representation of $A$ on $H_{0}$. The stability group 
$\mathfrak{U}_{0}$ of $\mathfrak{U}$ at $\pi_{0}$ is the unitary group of the commutant $\mathfrak{M}_{0}^{\prime}$ of $\mathfrak{T}_{0}$, so that $\mathfrak{U}_{0}$ is a closed subgroup. Therefore, the quotient map: $\mathfrak{U} \mapsto \mathfrak{U} / \mathfrak{U}_{0}$ has a Borel transversal $\mathfrak{B}$ by [3, Lemma 3]. Putting $\Psi(u)=u \pi_{0}$ for $u \in \mathfrak{U}, \Psi$ is a one-to-one Borel map of the standard Borel space $\mathfrak{B}$ into the standard Borel space $\operatorname{Rep}\left(A: H_{0}\right)$, so that it becomes a Borel isomorphism by [6, Theorem 3.2]. Let $\Phi$ denote the inverse map of $\Psi$ defined on $\Psi(\mathfrak{W})$. By the measurability of the map: $\gamma \mapsto u(\gamma) x u(\gamma)^{-1}$, $\gamma \mapsto u(\gamma) \pi_{0}$ is a $\operatorname{Rep}\left(A: H_{0}\right)$-valued measurable function over $\Gamma$, so that $\gamma \mapsto \Phi\left(u(\gamma) \pi_{0}\right)=v(\gamma)$ is also a measurable $\mathfrak{U}$-valued function on $\Gamma$. By the equality

$$
v(\gamma) \pi_{0}=\Psi(v(\gamma))=\Psi(u(\gamma)) \equiv u(\gamma) \pi_{0},
$$

$u(\gamma)^{-1} v(\gamma)$ belongs to $\mathfrak{U}_{0}$, so that $v(\gamma) x v(\gamma)^{-1}=u(\gamma) x u(\gamma)^{-1}$ for every $x \in \mathscr{N}_{0}$, which implies that $y_{i}(\gamma)=v(\gamma) x_{i} v(\gamma)^{-1}$ for every $\gamma$ of $\Gamma$ and $i \in I$. Putting

$$
v=\int_{\Gamma}^{\oplus} v(\gamma) d \mu(\gamma),
$$

$v$ becomes the desired isometry of $L^{2}(\Gamma, \mu) \otimes H_{0}$ onto $H$. This completes the proof.

Theorem 1 gives an affirmative answer to the question proposed by J. Dixmier in [1, p. 175].

TheOREM 2. Let $\mathfrak{M}_{0}$ be a von Neumann algebra acting on a separable Hilbert space $H_{0}$. Let $\gamma \rightarrow H(\gamma)$ be a measurable field of Hilbert spaces over $\Gamma$,

$$
H=\int_{\Gamma}^{\oplus} H(\gamma) d \mu(\gamma),
$$

a the algebra of all diagonal operators, $\gamma \mapsto \mathfrak{T}(\gamma) \subset ®(H(\gamma))$ a measurable field of von Neumann algebras and

$$
\mathfrak{N}=\int_{\Gamma}^{\oplus} \mathfrak{T}(\gamma) d \mu(\gamma) .
$$

Suppose that there exists, for every $\gamma \in \Gamma$, an isometry $u(\gamma)$ of $H_{0}$ onto $H(\gamma)$ such that $u(\gamma) \mathfrak{T}_{0} u(\gamma)^{-1}=\mathfrak{T}(\gamma)$. Then there exists an isometry of $L^{2}(\Gamma, \mu) \otimes H_{0}$ onto $H$ which transforms the tensor product $Q \otimes \mathfrak{T}_{0}$ into $\mathfrak{T}$.

Proof. We may assume that $\gamma \rightarrow H(\gamma)$ is a constant field corresponding to $H_{0}$. Then $u(\gamma)$ is a unitary operator on $H_{0}$ such that $u(\gamma) \mathscr{T}_{0} u(\gamma)^{-1}$ $=\mathfrak{N}(\gamma)$ for every $\gamma \in \Gamma$. Let $\mathfrak{A}$ denote the set of all von Neumann algebras acting on $H_{0}$. By [4], $\mathfrak{A}$ has a standard Borel structure with 
the property: $\boldsymbol{\gamma} \rightarrow \mathfrak{T}(\boldsymbol{\gamma}) \in \mathfrak{A}$ is a measurable field if and only if $\boldsymbol{\gamma} \rightarrow \mathfrak{T}(\boldsymbol{\gamma})$ is a $\mathfrak{A}$-valued measurable function over $\Gamma$. Define the action of the unitary group $\mathfrak{U}$ on $\mathfrak{A}$ by $u(\mathfrak{T})=u \mathfrak{T}\left(u^{-1}\right.$ for $u \in \mathfrak{U}$ and $\mathfrak{T} \in \mathfrak{A}$. Then $\mathfrak{U}$ becomes a Borel transformation group of $\mathfrak{U}$ by [5]. Let $\mathfrak{U}_{0}$ denote the stability group of $\mathfrak{U}$ at $\mathfrak{T}_{0}$. Then $\mathfrak{U}_{0}$ is a closed subgroup of $\mathfrak{u}$. Therefore, the quotient map: $\mathfrak{U} \mapsto \mathfrak{u} / \mathfrak{U}_{0}$ has a Borel transversal $\mathfrak{W}$ as in Theorem 1. Putting $\Psi(u)=u\left(\mathfrak{T}_{0}\right)$ for $u \in \mathfrak{U}, \Psi$ becomes a one-toone Borel map of the standard Borel space $\mathfrak{W}$ into the standard Borel space $\mathfrak{A}$, so that $\Psi$ is a Borel isomorphism of $\mathfrak{W}$ onto $\Psi(\mathfrak{W})$. Let $\Phi$ denote the inverse map of $\Psi$ defined on $\Psi(\mathfrak{B})$. Putting $v(\gamma)=\Phi(\mathfrak{T H}(\gamma))$ for every $\gamma \in \Gamma, \gamma \rightarrow v(\gamma)$ is a measurable $\mathfrak{U}$-valued function over $\Gamma$. Then

$$
v=\int_{\Gamma}^{\oplus} v(\gamma) d \mu(\gamma)
$$

is the desired isometry of $L^{2}(\Gamma, \mu) \otimes H_{0}$ onto $H$. This completes the proof.

Making use of Theorem 2, we can settle the problem proposed by S. Sakai in [7, Problem 4, p. 3.55] as follows:

TheOREM 3. Let $\gamma \rightarrow \mathfrak{M}(\gamma)$ be a measurable field of von Neumann algebras over $\Gamma$. Suppose each von Neumann algebra $\mathfrak{T}(\gamma)$ is isomorphic to a fixed von Neumann algebra $\mathfrak{M}_{0}$. Then the direct integral

$$
\Re=\int_{\Gamma}^{\oplus} \mathscr{T}(\gamma) d \mu(\gamma)
$$

is isomorphic to the tensor product $Q \otimes M_{0}$ of the diagonal algebra $Q$ and $\mathfrak{M T}_{0}$.

Proof. Suppose $\gamma \mapsto H(\gamma)$ is a measurable field of Hilbert spaces over $\Gamma$ associated with $\gamma \mapsto \mathfrak{T C}(\gamma)$; that is, each $\mathfrak{T}(\gamma)$ acts on $H(\gamma)$. Let $K_{0}$ be a countably infinite dimensional Hilbert space. Then $\gamma \mapsto H(\gamma) \otimes K_{0}=\widetilde{H}(\gamma)$ is a measurable field of Hilbert spaces by $[1$, Proposition 11, p. 152]. Further, $\gamma \mapsto \mathscr{T}(\gamma) \otimes I=\widetilde{\mathscr{N}}(\gamma)$ also becomes a measurable field of von Neumann algebras. Let $H_{0}$ denote the underlying Hilbert space of $\mathfrak{T}_{0}$. Putting $\tilde{\mathfrak{T}}_{0}=\mathfrak{N}_{0} \otimes I$ on $\tilde{H}_{0}=H_{0} \otimes K_{0}$, each $\tilde{\mathfrak{N}}(\gamma)$ is isomorphic to $\tilde{\mathfrak{N}}_{0}$. Since the commutant $\tilde{\mathfrak{N}}(\gamma)^{\prime}$ of each $\tilde{\mathscr{N}}(\gamma)$ becomes $\mathscr{T}(\gamma)^{\prime} \otimes B\left(K_{0}\right)$ which is properly infinite, each algebra $\tilde{\mathscr{N}}(\gamma)$ is spatially isomorphic to $\tilde{\mathfrak{N}}_{0}^{-}$. Therefore, by Theorem 2 , the direct integral

$$
\tilde{\mathfrak{N}}=\int_{\Gamma}^{\oplus} \tilde{\mathfrak{T}}(\gamma) d \mu(\gamma)
$$


is spatially isomorphic to $a \otimes \tilde{\mathfrak{N}}_{0}$. By [1, Proposition 3, p. 185], $\tilde{\mathfrak{N}}$ and $a \otimes \tilde{N}_{0}$ are spatially isomorphic to $\mathscr{T} \otimes I$ and $\left(a \otimes \mathscr{N}_{0}\right) \otimes I$ respectively, so that $\mathscr{N}$ is isomorphic to $a \otimes \mathfrak{N}_{0}$. This completes the proof.

\section{REFERENCES}

1. J. Dixmier, Les algebres d'operateurs dans l'espace hilbertien, Gauthier-Villars, Paris, 1957.

2. - Les $C^{*}$-algebres et leurs representations, Gauthier-Villars, Paris, 1964.

3. - Dual et quasi-dual d'une algèbre de Banach involutive, Trans. Amer. Math. Soc 104 (1962), 278-283.

4. E. G. Effros, The Borel space of von Neumann algebras on a separable Hilbert space, Pacific J. Math. 15 (1965), 1153-1164.

5. - Global structure in von Neumann algebras, Trans. Amer. Math. Soc. 121 (1966), 434-454.

6. G. W. Mackey, Borel structure in groups and their duals, Trans. Amer. Math. Soc. 85 (1957), 134-165.

7. S. Sakai, The theory of $W^{*}$-algebras, Mimeographed note, Yale Univ., New Haven, Conn., 1962.

TôHoku University, SENDAI, Japan 